\title{
A Local Solution to the Output Regulation Problem for Sampled-Data Systems on Commutative Matrix Lie Groups
}

\author{
Philip James McCarthy* Christopher Nielsen ${ }^{\dagger}$
}

\begin{abstract}
We present a smooth nonlinear control law for a kinematic plant on commutative matrix Lie groups that achieves regulation, if the state tracking and estimation errors are initialized in a suitable neighbourhood of identity. We show that in exponential coordinates, the closed-loop dynamics are linear. Our control law uses output feedback; to this end, we propose an almost-globally defined state estimator.
\end{abstract}

\section{INTRODUCTION}

We examine the regulator problem for systems that evolve on matrix Lie groups, using a sampled-data feedback control configuration. The regulator problem is one of the central problems in control theory. It combines stabilization with reference tracking and disturbance rejection. The regulator problem has been analyzed in great detail in the linear case [1] and the nonlinear case, in both continuous- [2] and discrete-time [3]. More recently, the continuous-time regulator problem for systems on Lie groups has received some attention. Almost-global output regulation was achieved for a class of systems evolving on $\mathrm{SE}(n)$ by identifying a separation principle and using output feedback [4]. Local output regulation was also achieved for a class of systems evolving on general Lie groups with outputs in a homogeneous vector space and exostates evolving on a compact set [5].

There are many dynamical systems whose state spaces are naturally modelled as matrix Lie groups. Networks of oscillators can be modelled on $\mathrm{SO}(2)^{n}$ [6]. The group $\mathrm{SE}(3)$ captures the dynamics of rigid bodies in space, such as underwater vehicles [7] and UAVs [8]. Planar motion of robots can be modelled on $\mathrm{SE}(2)$ [9]. Quantum systems evolve on the unitary groups $\mathrm{U}(n)$ and $\mathrm{SU}(n)$ [10]. Even the noise responses of some circuits evolve on Lie groups [11].

In general, Lie groups are not vector spaces; they are smooth manifolds endowed with a group structure, which facilitates control design in global coordinates as well as local design and analysis using the rich theory of control on manifolds. The Lie structure has been leveraged, for example, for motion tracking on $\mathrm{SE}(3)$ [12], and the control of UAV [13] and spacecraft [14] orientation on SO(3).

We study such systems in the sampled-data configuration, i.e., a continuous-time plant and a discrete-time controller. This configuration is ubiquitous in applied control [15], where controllers are implemented on embedded devices.

\footnotetext{
* Supported by the Ontario Graduate Scholarship (OGS).

† Supported by the Natural Sciences and Engineering Research Council of Canada (NSERC).

The authors are with the Dept. of Electrical and Computer Engineering, University of Waterloo, Waterloo ON, N2L 3G1 Canada. \{philip. mccarthy; cnielsen\} @uwaterloo.ca
}

The main sampled-data design approach in practice is emulation. A continuous-time controller is designed for the continuous-time plant, but is implemented using an approximate discretization. If the sampling-period is sufficiently small, then the actual closed-loop system is stable. This technique has two key shortcomings [16]: 1) it may not be possible for a given approximate discretization method, e.g., Euler's method; 2) it relies on fast sampling, which may not be possible due to hardware limitations. For example, a UAV's translation relative to a reference can be computed using machine vision, where the speed of sampling is limited by the frame rate of the camera, e.g., $25 \mathrm{~Hz}$ [17].

For LTI systems, the continuous-time state trajectory can be solved exactly, thereby admitting a discrete-time model that matches the continuous-time behaviour at the sampling instants. This enables analysis and design to be done entirely in discrete-time, hence the controller can be implemented exactly. This technique is called direct design. Its key advantage is that it allows guarantees about performance to be made at the sampling instants, such as stability. This is not the case for most nonlinear systems, which do not admit exact discretizations. The efficacy of direct design depends on the accuracy of the discretization. In one case study, sampling periods as low as $30 \mu$ s, combined with an Euler discretization of a synchronous machine plant, yielded an ineffectual model predictive controller [15]. Right (left)invariant systems on matrix Lie groups are an exception, in that for piecewise constant inputs, the state trajectories have exact solutions [18].

Sampled-data control of systems on Lie groups has heretofore been subject to limited formal study, including the authors' works on passivity [19] and synchronization [20] on matrix Lie groups. Controllability using multirate piecewise constant inputs was investigated for matrix Lie groups in [21] and control-affine systems whose Lie algebra of vector fields is nilpotent in [22]. Discrete-time control has also received some attention [23]. The closely related class of bilinear systems has also been studied in the discrete-time [18] and sampled-data [24] settings.

We present a control law that solves the regulator problem for sampled-data systems on commutative matrix Lie groups. If the state tracking and estimation errors are initialized in a suitable neighbourhood of identity, then regulation is achieved.

\section{A. Notation and Terminology}

For $n \in \mathbb{N}$, let $\mathbb{N}_{n}:=\{1, \ldots, n\}$. Given a matrix $M \in \mathbb{C}^{m \times n}, M^{\top}$ is its (non-Hermitian) transpose, $\|M\|$ is 
its Frobenius norm, $M^{\dagger}$ is its Moore-Penrose pseudoinverse, and $\operatorname{vec}(M):=\left[\begin{array}{llllll}M_{11} & \cdots & M_{m 1} & M_{21} & \cdots & M_{m n}\end{array}\right]^{\top} \in$ $\mathbb{C}^{m n}$; if $m=n$, then $\sigma(M)$ is its spectrum. Given an ordered set of matrices $\left\{M_{1}, \ldots, M_{q}\right\}$, define $\bar{M}:=$ $\left[\operatorname{vec}\left(M_{1}\right) \quad \cdots \quad \operatorname{vec}\left(M_{q}\right)\right] \in \mathbb{C}^{m n \times q}$. Let $\mathbf{0}_{n} \in \mathbb{R}^{n}$ and $\mathbf{0}_{m \times n} \in \mathbb{R}^{m \times n}$ denote the column and matrix of zeros, respectively. When a discrete-time signal appears in a continuous-time expression, it to be understood as having passed through an ideal zero order hold.

\section{Sampled-Data Regulator Problem}

We consider a plant modelled by the differential equation

$$
\dot{X}=\left(A+\sum_{i=1}^{m} B_{i} u_{i}+\sum_{i=1}^{r_{d}} Q_{d i} w_{d i}+\sum_{i=1}^{r_{c}} Q_{c i} w_{c i}\right) X
$$

with measured output

$$
Y=\exp \left(C+\sum_{i=1}^{r_{d}} D_{d i} w_{d i}+\sum_{i=1}^{r_{c}} D_{c i} w_{c i}\right) X
$$

We assume, as is typical, that the exogenous signals $w_{d}, w_{c}$ evolve according to known dynamics, modelled as

$$
w_{d}^{+}=S_{d} w_{d}, \quad \dot{w}_{c}=S_{c} w_{c} .
$$

Here, $X \in \mathrm{G}$ where $\mathrm{G} \subset \mathrm{GL}(N, \mathbb{C})$ is an $n$-dimensional connected matrix Lie group over the complex field $\mathbb{C}$ which includes, as a special case, real matrix Lie groups. The matrices $A, B_{i}, Q_{c i}, Q_{d i}, C, D_{d i}$, and $D_{c i}$ are elements of the Lie algebra $\mathfrak{g}$ of $\mathrm{G}$, which is a vector space over a field $\mathbb{F}$ equal to either $\mathbb{C}$ or $\mathbb{R}$. The control input is $u \in \mathbb{F}^{m}$, the discrete- and continuous-time exostates are $w_{d} \in \mathbb{R}^{r_{d}}$ and $w_{c} \in \mathbb{R}^{r_{c}}$, respectively, and $S_{d} \in \mathbb{R}^{r_{d} \times r_{d}}, S_{c} \in \mathbb{R}^{r_{c} \times r_{c}}$.

Assumption 1. The Lie group $\mathrm{G}$ is commutative.

Assumption 1 is restrictive, as all commutative matrix Lie groups are diffeomorphic to $\mathbb{R}^{k} \times \mathbb{T}^{n-k}$, as discussed in Section III-B. We examine this class of Lie groups to facilitate this preliminary research in this area. However, there are systems of practical interest on such Lie groups, e.g., networks of oscillators on $\mathrm{SO}(2)^{n} \cong \mathbb{T}^{n}[6]$ and singleaxis rigid body manoeuvres on $\mathbb{R} \times S O(2) \cong \mathbb{R} \times \mathbb{T}$ [25].

Assumption 2. The spectra of $S_{d}$ and $S_{c}$ lie outside the open unit disc and in the closed right half plane, respectively.

Assumption 2 is not restrictive. If $S_{d}$ or $S_{c}$ has stable eigenvalues, then the dynamics of (3) can be redefined as their restriction to the unstable modal subspaces [26, Chapter $2, \S 3$ ] of $S_{d}$ and $S_{c}$. The dynamics on the stable modal subspaces can be ignored, since tracking or rejecting a zero signal is equivalent to stability.

Equation (1) is a kinematic model of a system evolving on a matrix Lie group G, where the output (2) models the information that is available for feedback. The exosystem (3) comprises both discrete- and continuous-time subsystems. This enables modelling of, for example, physical plants that are subject to continuous-time disturbances, but are sent reference signals from a computer. The plant is assumed to be fully actuated in the sense that $\operatorname{span}_{\mathbb{F}}\left\{B_{1}, \ldots, B_{m}\right\}=\mathfrak{g}$. We justify this assumption in Section $\mathrm{V}$-A. We are interested in the sampled-data control of this system in which the control law is implemented on an embedded computer, which we explicitly model using the setup in Figure 1 . The blocks $H$ and $S$ in Figure 1 are the ideal hold and sample operators, respectively. Sample and hold are, respectively, idealized models of $\mathrm{A} / \mathrm{D}$ and $\mathrm{D} / \mathrm{A}$ conversion.

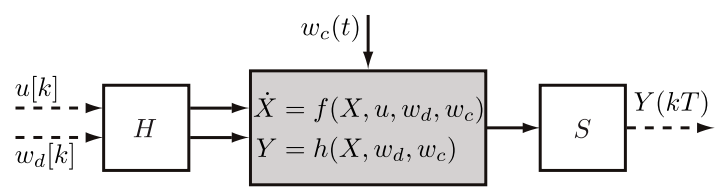

Fig. 1: Sampled-data plant on a matrix Lie group G.

Assumption 3. The sample and hold blocks operate at the same period $T>0$ and are synchronized.

Under Assumption 3, letting $X[k]:=X(k T), u[k]:=$ $u(k T)$, and $w_{c}[k]:=w_{c}(k T)$, the plant (1) and exosystem (3) have exact discretizations, as we will prove in Section IV:

$$
\begin{gathered}
X^{+}=\exp \left(T A+T \sum_{i=1}^{m} B_{i} u_{i}+T \sum_{i=1}^{r_{d}} Q_{d i} w_{d i}\right. \\
\left.+\sum_{i=1}^{r_{c}} Q_{c i} \mathrm{e}_{i}^{\top} \int_{0}^{T} e^{\tau S_{c}} \mathrm{~d} \tau w_{c}\right) X,
\end{gathered}
$$

where $\mathrm{e}_{i}$ is the $i$ th canonical basis vector of $\mathbb{R}^{r_{c}}$, and

$$
\left[\begin{array}{c}
w_{d}^{+} \\
w_{c}^{+}
\end{array}\right]=\underbrace{\left[\begin{array}{cc}
S_{d} & \mathbf{0}_{r_{d} \times r_{c}} \\
\mathbf{0}_{r_{c} \times r_{d}} & e^{T S_{c}}
\end{array}\right]}_{S} \underbrace{\left[\begin{array}{l}
w_{d} \\
w_{c}
\end{array}\right]}_{w} .
$$

\section{A. The Sampled-Data Regulator Problem}

The goal of the regulator problem is to drive a regulation quantity to identity. Define the regulation quantity

$$
Z=\exp \left(F+\sum_{i=1}^{r_{d}} G_{d i} w_{d i}+\sum_{i=1}^{r_{c}} G_{c i} w_{c i}\right) Y
$$

where $F, G_{d i}, G_{c i} \in \mathfrak{g}$.

Local Output Regulation on Matrix Lie Groups : Given a plant with continuous-time dynamics (1), output (2), regulation quantity (6), sampling period $T>0$, and exosystem (3), find, if possible, a discrete-time control law $u$, which depends on only the output $Y$, such that for all initial conditions $X(0), w_{c}(0), w_{d}[0]$, in a neighbourhood of the identity in $\mathrm{G} \times \mathbb{R}^{r_{d}} \times \mathbb{R}^{r_{c}}, Z[k] \rightarrow I_{N}$ as $k \rightarrow \infty$.

Such a $u$ is said to solve the regulator problem with output information. If instead the input $u$ has access to $X, w_{d}$, and $w_{c}$, then $u$ is said to solve the the regulator problem with full information. We will first solve the latter using a controller of the form $u:=\Gamma\left(X, w_{d}, w_{c}\right)+$ $\Psi\left(w_{d}, w_{c}\right)$. We then extend our result to design an output feedback controller using a dynamic state estimator. 


\section{PRELIMINARIES}

\section{A. The matrix logarithm}

Theorem III.1 ([27, Theorem 1.31]). If $X \in \mathbb{F}^{n \times n}$ has no nonpositive real eigenvalues, then there exists a unique $A \in$ $\mathbb{F}^{n \times n}$, whose spectrum lies in $\{z \in \mathbb{C}:-\pi<\operatorname{Im}(z)<\pi\}$, such that $\exp (A)=X$.

The matrix $A$ in Theorem III.1 is the principal logarithm of $X$ and is denoted by $\log (X)$. If $\|X-I\|<1$, then $\log (X)=\sum_{k=1}^{\infty} \frac{(-1)^{k-1}}{k}(X-I)^{k}$. The principal logarithm is the inverse of the matrix exponential, but only on a subset $U \subset \mathrm{GL}(N, \mathbb{C})$ containing the identity. For example, on $\mathrm{GL}(N, \mathbb{C}), \log$ is well-defined on $U=$ $\{\exp (A) \in \mathrm{GL}(N, \mathbb{C}):\|A\|<\log (2)\}$, but on $\mathrm{SO}(n), U=$ $\{R \in \mathrm{SO}(n):-1 \notin \sigma(R)\}$, even though $\|R\|=\sqrt{n}$ for all $R \in \mathrm{SO}(n)$.

\section{B. Exponential Coordinates and One-Parameter Subgroups}

Definition III.2 (One-Parameter Subgroup). Given a Lie group G, a one-parameter subgroup is a continuous morphism of groups $\phi: \mathbb{R} \rightarrow \mathrm{G}$.

To generalize the concept of one-parameter subgroups to higher dimensional manifolds, we consider generalized cylinders. A generalized cylinder is an $n$-dimensional manifold that is diffeomorphic to $\mathbb{T}^{k} \times \mathbb{R}^{n-k}$. Such a diffeomorphism exists if and only if there exist $n$ commutative and everywhere-linearly-independent vector fields on the manifold [28, §49]. If the manifold is a Lie group $G$, then this simplifies to the requirement that its Lie algebra $\mathfrak{g}$ have a commutative basis.

Let $G$ be such a manifold and fix a commutative basis $H_{1}, \ldots, H_{n}$ for its Lie algebra $\mathfrak{g}$. Consider the one-parameter groups $\phi_{i}: \mathbb{R} \rightarrow \mathrm{G}$ associated with each $H_{i}$. The image of $\phi\left(x_{1}, \ldots, x_{n}\right):=\phi_{1}\left(x_{1}\right) \phi_{2}\left(x_{2}\right) \cdots \phi_{n}\left(x_{n}\right)$ is $\mathrm{G}$. Without loss of generality, let $\phi_{i}, i \in \mathbb{N}_{k}, 0 \leq k \leq n$ have nonzero kernel, and let $\phi_{i}, i \in\{k+1, \ldots, n\}$ have zero kernel.

Fixing such a basis, the Log map induces local coordinates on $\mathrm{G} \cap U$. Given $X \in \mathrm{G}$, by commutativity of $H_{1}, \ldots, H_{n}$, $X=\exp \left(x_{1} H_{1}\right) \cdots \exp \left(x_{n} H_{n}\right)=\exp \left(x_{1} H_{1}+\cdots+x_{n} H_{n}\right)$. If $X \in \mathrm{G} \cap U$, then $\log (X)=x_{1} H_{1}+\cdots+x_{n} H_{n}$. Then, by linear independence of $H_{1}, \ldots, H_{n}, x_{1}, \ldots, x_{n}$ can be uniquely determined, yielding local coordinates $\left(x_{1}, \ldots, x_{n}\right) \in \mathbb{R}^{n}$. Thus, a Lie group $\mathrm{G}$ can be locally identified with an open subset of the vector space $\mathbb{R}^{n}$ containing the origin. By commutativity of $\mathrm{G}$, these local coordinates coincide with the familiar exponential coordinates of both the first and second kinds [29, Remarks 5.33.1].

\section{Properties of the composed flow}

The map $\phi: \mathbb{R}^{n} \rightarrow \mathrm{G}$, defined in the previous section, is critical to our analysis throughout this paper. In this section, we establish important properties of $\phi$ when $G$ is a generalized cylinder.

Proposition III.3. If $\mathrm{G}$ is a generalized cylinder, then $\phi$ : $\mathbb{R}^{n} \rightarrow \mathrm{G}$ is a morphism of groups.
Proof. Let $x_{i}=\left(x_{i}^{(1)}, \ldots, x_{i}^{(n)}\right) \in \mathbb{R}^{n}$ and $x_{j}=\left(x_{j}^{(1)}, \ldots, x_{j}^{(n)}\right) \in \mathbb{R}^{n}$, where $\phi\left(x_{i}\right)=X_{i}$ and $\phi\left(x_{j}\right)=X_{j}$. By commutativity of $H_{1}, \ldots, H_{n}$, $\phi\left(x_{i}+x_{j}\right)=\prod_{k=1}^{n} \exp \left(\left(x_{i}^{(k)}+x_{j}^{(k)}\right) H_{k}\right)=$ $\prod_{k=1}^{n} \exp \left(x_{i}^{(k)} H_{k}\right) \prod_{k=1}^{n} \exp \left(x_{j}^{(k)} H_{k}\right)$ $\phi\left(x_{i}\right) \phi\left(x_{j}\right)$.

To facilitate discussion and greatly simplify presentation, we shall tacitly make extensive use of the following simple lemma, whose proof follows from direct computation.

Lemma III.4. Let $p, q \in \mathbb{N}, A_{1}, \ldots, A_{p} \in \mathbb{C}^{q \times q}$, and $\xi \in$ $\mathbb{C}^{p}$. Then $\sum_{k=1}^{p} A_{k} \xi_{k}=\operatorname{vec}^{-1}(\bar{A} \xi)$.

When $\mathrm{G}$ is a matrix Lie group, the map $\phi$ is given by $\phi(x)=\exp \left(\operatorname{vec}^{-1}(\bar{H} x)\right)$.

Proposition III.5. In a neighbourhood of identity $U \subset \mathrm{G}$, $\phi: \mathbb{R}^{n} \rightarrow \mathrm{G}$ has inverse

$$
\phi^{-1}(X)=\bar{H}^{\dagger} \operatorname{vec}(\log (X)) .
$$

Proof. Since $\bar{H}: \mathbb{R}^{n} \rightarrow \operatorname{vec}(\mathfrak{g})$ is injective, a left inverse is $\bar{H}^{\dagger}: \operatorname{vec}(\mathfrak{g}) \rightarrow \mathbb{R}^{n}$, which is surjective. It follows from direct computation that (7) is a left inverse of $\phi$.

To prove that (7) is a right inverse of $\phi$, we require $\bar{H}^{\dagger}$ to be a right inverse of $\bar{H}$, which does not hold globally. Let $\bar{H}^{-1} \operatorname{vec}(\mathfrak{g}):=\left\{x \in \mathbb{R}^{n}: \bar{H} x \in \operatorname{vec}(\mathfrak{g})\right\}$ be the preimage of $\operatorname{vec}(\mathfrak{g})$ under $\bar{H}$, and let $\bar{H}^{\dagger} \mid \bar{H}^{-1} \operatorname{vec}(\mathfrak{g})$ be the restriction of $\bar{H}^{\dagger}$ to this subspace. Let $x \in \mathbb{R}^{n}$, then $\bar{H} \bar{H}^{\dagger}(\bar{H} x)=$ $\bar{H}\left(\bar{H}^{\dagger} \bar{H}\right) x=\bar{H} x$. Since two-sided inverses are unique, this implies that on the domain $\bar{H}^{-1} \operatorname{vec}(\mathfrak{g})$, the left inverse of $\bar{H}$, $\bar{H}^{\dagger}$, is also the right inverse. Therefore, $\bar{H} \bar{H}^{\dagger} \mid \bar{H}^{-1} \operatorname{vec}(\mathfrak{g})$ is identity. The rest follows from direct computation.

Proposition III.6. The map $\phi^{-1}: U \subset \mathrm{G} \rightarrow \mathbb{R}^{n}$ defined in (7) is a morphism of groups.

Proof. The inverse of a morphism, if it exists, is itself a morphism [30, Chapter 1, Theorem 20].

\section{LOCAL DYNAMICS}

Proposition IV.1. The continuous-time plant (1) has exact discretization (4).

Proof. Consider a matrix ODE $\dot{X}(t)=M(t) X(t)$. If for all $t, t^{\prime} \geq 0, M(t) M\left(t^{\prime}\right)=M\left(t^{\prime}\right) M(t)$, then for $t \geq 0$, $X(t)=\int_{0}^{t} M(\tau) \mathrm{d} \tau X(0)[31, \S \mathrm{V}]$. Since $\mathfrak{g}$ is commutative, the vector field commutation property holds for all time. Solve (1) at $t=k T$ and $t=(k+1) T$. Factoring the former solution from the latter yields (4).

Much of our analysis is facilitated by using local coordinates. Define $\widetilde{A}:=\bar{H}^{\dagger} \bar{A}, \widetilde{C}:=\bar{H}^{\dagger} \bar{C}, \widetilde{F}:=\bar{H}^{\dagger} \bar{F}$, $\widetilde{Q}_{d}:=\bar{H}^{\dagger} \bar{Q}_{d}$, and $\widetilde{Q}_{c}:=\bar{H}^{\dagger} \bar{Q}_{c} \int_{0}^{T} e^{\tau S_{c}} \mathrm{~d} \tau, \widetilde{D}_{d}:=\bar{H}^{\dagger} \bar{D}_{d}$, $\widetilde{D}_{c}:=\bar{H}^{\dagger} \bar{D}_{c}, \widetilde{G}_{d}:=\bar{H}^{\dagger} \bar{G}_{d}$, and $\widetilde{G}_{c}:=\bar{H}^{\dagger} \bar{G}_{c}$. For $X, Y, Z \in U$, we compute $y:=\phi^{-1}(Y), z:=\phi^{-1}(Z)$, 
and the discrete-time dynamics of $x:=\phi^{-1}(X)$ :

$$
\begin{aligned}
x^{+} & =T \widetilde{A}+T \widetilde{B} u+T \widetilde{Q}_{d} w_{d}+\widetilde{Q}_{c} w_{c}+x \\
y & =\widetilde{C}+\widetilde{D}_{d} w_{d}+\widetilde{D}_{c} w_{c}+x, \\
z & =(\widetilde{F}+\widetilde{C})+\left(\widetilde{G}_{d}+\widetilde{D}_{d}\right) w_{d}+\left(\widetilde{G}_{c}+\widetilde{D}_{c}\right) w_{c}+x .
\end{aligned}
$$

\section{Controllability and State Estimation}

Our solution to the regulator problem uses output feedback. Thus, we require local detectability and stabilizability.

\section{A. Controllability}

We first define and characterize the notion of controllability for a discrete-time linear-affine system:

$$
x^{+}=A x+B u+c,
$$

where $A \in \mathbb{R}^{n \times n}, B \in \mathbb{R}^{n \times m}, x, c \in \mathbb{R}^{n}$, and $u \in \mathbb{R}^{m}$.

Definition V.1. System (9) is controllable if for all $x_{0}, \bar{x} \in$ $\mathbb{R}^{n}$, given $x[0]=x_{0}$, there exist $k \in \mathbb{Z}_{\geq 0}$ and $u[0], \ldots, u[k-1] \in \mathbb{R}^{m}$ such that $x[k]=\bar{x}$.

Proposition V.2. System (9) is controllable if and only if the pair $(A, B)$ is controllable.

Proof. Routine calculation verifies $x[n]=A^{n} x[0]+$ $\sum_{i=0}^{n-1} A^{i} B u[n-1-i]+\sum_{i=0}^{n-1} A^{i} c$. Thus, driving system (9) to $\bar{x} \in \mathbb{R}^{n}$ in $n$ time steps is equivalent to driving the linear system $x^{+}=A x+B u$ to $\bar{x}-\sum_{i=0}^{n-1} A^{i} c$ in $n$ time steps. This is possible if and only if $(A, B)$ is controllable.

We now analyze the controllability of the sampled plant (4). Set the exostates $w_{d}$ and $w_{c}$ to zero. Then the local sampled dynamics (8) reduce to $x^{+}=T \widetilde{A}+T \widetilde{B} u+x$.

Proposition V.3. The sampled plant (4) is controllable if and only if $\operatorname{span}_{\mathbb{F}}\left\{B_{1}, \ldots, B_{m}\right\}=\mathfrak{g}$.

Proof. In local coordinates (8), the state matrix is identity, so the PBH test for controllability reduces to $\operatorname{rank}(\widetilde{B})=n$. This is satisfied if and only if $\operatorname{span}_{\mathbb{F}}\left\{B_{1}, \ldots, B_{m}\right\}=\mathfrak{g}$.

\section{B. State Estimation}

Let $L_{1} \in \mathbb{R}^{n \times n}, L_{2} \in \mathbb{R}^{r_{d} \times n}, L_{3} \in \mathbb{R}^{r_{c} \times n}$, $\widehat{X}, \widehat{Y} \in \mathrm{G}, \quad \hat{w}_{d} \in \mathbb{R}^{r_{d}}, \quad \hat{w}_{c} \in \mathbb{R}^{r_{c}}, \quad e_{y}:=$ $\phi^{-1}\left(\widehat{Y} Y^{-1}\right)$. Defining the output estimate $\widehat{Y}=$ $\exp \left(C+\sum_{i=1}^{r_{d}} D_{d i} \hat{w}_{d i}+\sum_{i=1}^{r_{c}} D_{c i} \hat{w}_{c i}\right) \widehat{X}$, we propose a state estimator with dynamics:

$$
\begin{aligned}
\widehat{X}^{+}= & \exp \left(T A+T \sum_{i=1}^{m} B_{i} u_{i}+T \sum_{i=1}^{r_{d}} Q_{d i} \hat{w}_{d i}\right. \\
& \left.+\sum_{i=1}^{r_{c}} Q_{c i} \mathrm{e}_{i}^{\top} \int_{0}^{T} e^{\tau S_{c}} \mathrm{~d} \tau \hat{w}_{c}+\operatorname{vec}^{-1}\left(\bar{H} L_{1} e_{y}\right)\right) \widehat{X} \\
\hat{w}_{d}^{+}= & S_{d} \hat{w}_{d}+L_{2} e_{y} \\
\hat{w}_{c}^{+}= & e^{T S_{d}} \hat{w}_{c}+L_{3} e_{y} .
\end{aligned}
$$

Given the output $Y$, we seek to choose $L_{1}, L_{2}$, and $L_{3}$, such that the estimation errors $E_{x}:=\widehat{X} X^{-1} \rightarrow I_{N}, e_{w_{d}}:=$ $\hat{w}_{d}-w_{d} \rightarrow \mathbf{0}_{r_{d}}$, and $e_{w_{c}}:=\hat{w}_{c}-w_{c} \rightarrow \mathbf{0}_{r_{c}}$ as $k \rightarrow \infty$.
In local coordinates, the output estimation error $e_{y}$ and the dynamics of the state estimation error $e_{x}:=\phi^{-1}\left(E_{x}\right)$ are $e_{y}=\widetilde{D}_{c} e_{w_{c}}+\widetilde{D}_{d} e_{w_{d}}+e_{x}$ and $e_{x}^{+}=e_{x}+T \widetilde{Q}_{d} e_{w_{d}}+$ $\widetilde{Q}_{c} e_{w_{c}}+L_{1} e_{y}$, from which, direct calculation verifies

$$
\begin{aligned}
{\left[\begin{array}{c}
e_{x}^{+} \\
e_{w_{d}}^{+} \\
e_{w_{c}}^{+}
\end{array}\right]=} & \overbrace{\left[\begin{array}{ccc}
I_{n} & T \widetilde{Q}_{d} & \widetilde{Q}_{c} \\
\boldsymbol{0}_{r_{d} \times n} & S_{d} & \mathbf{0}_{r_{d} \times r_{c}} \\
\mathbf{0}_{r_{c} \times n} & \mathbf{0}_{r_{c} \times r_{d}} & e^{T S_{c}}
\end{array}\right]}^{A_{o}} \\
+ & \underbrace{\left[\begin{array}{c}
L_{1} \\
L_{2} \\
L_{3}
\end{array}\right]}_{L} \underbrace{\left[\begin{array}{ccc}
I_{n} & \widetilde{D}_{d} & \widetilde{D}_{c}
\end{array}\right]}_{C_{o}})\left[\begin{array}{c}
e_{x} \\
e_{w_{d}} \\
e_{w_{c}}
\end{array}\right],
\end{aligned}
$$

which are the estimation error dynamics of a Luenberger observer. Thus, we directly apply linear observer theory. Since $A_{o}$ is block diagonal, we have $\sigma\left(A_{o}\right)=\sigma\left(I_{n}\right) \sqcup$ $\sigma\left(S_{d}\right) \sqcup \sigma\left(e^{T S_{c}}\right)$. Thus, under Assumption 2, $\sigma\left(A_{0}\right)$ is entirely outside the open unit disc, so $\left(C_{o}, A_{o}\right)$ is detectable if and only if it is observable.

Proposition V.4. The pair $\left(C_{o}, A_{o}\right)$ is observable if and only if the following matrix is full rank:

$$
\left[\begin{array}{cc}
\widetilde{D}_{d}\left(S_{d}-I_{r_{d}}\right)+T \widetilde{Q}_{d} & \widetilde{D}_{c}\left(e^{T S_{c}}-I_{r_{c}}\right)+\widetilde{Q}_{c} \\
\vdots & \vdots \\
\widetilde{D}_{d}\left(S_{d}^{n+r_{d}+r_{c}}-I_{r_{d}}\right) & \widetilde{D}_{c}\left(\left(e^{T S_{c}}\right)^{n+r_{d}+r_{c}}-I_{r_{c}}\right) \\
+T \widetilde{Q}_{d} \sum_{i=0}^{n+r_{d}+r_{c}-1} S_{d}^{i} & +\widetilde{Q}_{c} \sum_{i=0}^{n+r_{d}+r_{c}}\left(e^{T S_{c}}\right)^{i}
\end{array}\right] .
$$

Proof. Given the observability matrix of $\left(C_{o}, A_{o}\right)$, subtracting the top block row from those below yields a block triangular matrix with block diagonal elements $I_{n}$ and (11).

Corollary V.5. If $\left(C_{o}, A_{o}\right)$ is observable, then the following are equivalent:

1) there exists an $L$ such that $A_{o}+L C_{o}$ is Schur;

2) the matrix (11) is full rank;

3) the point $\left(E_{x}, e_{w_{d}}, e_{w_{c}}\right)=\left(I_{N}, \mathbf{0}_{r_{d}}, \mathbf{0}_{r_{c}}\right)$ is locally asymptotically stable.

Thus, for suitable $L$ and initial conditions, the state estimator dynamics (10) are well-defined for all $k \geq 0$.

\section{Vi. Solution to the Regulator Problem}

In this section, we propose a solution to the regulator problem using state feedback. We also prove a separation principle, which allows the state feedback controller and state estimator to be designed independently. As with linear systems, this yields an output feedback controller that solves the regulator problem.

\section{A. Regulation with Full Information}

We design our controller in two parts: 1) a map $\Psi: \mathbb{R}^{r_{d}} \times$ $\mathbb{R}^{r_{c}} \cong \mathbb{R}^{r_{d}+r_{c}} \rightarrow \mathbb{F}^{m}$ that makes the manifold $\{X \in \mathrm{G}$ : $\left.Z=I_{N}\right\}$ invariant; 2) a state feedback $K: \mathbb{R}^{n} \rightarrow \mathbb{F}^{m}$ that renders this manifold locally asymptotically stable. 
We first find the state reference $\Pi: \mathbb{R}^{r_{d}} \times \mathbb{R}^{r_{c}} \cong$ $\mathbb{R}^{r_{d}+r_{c}} \rightarrow \mathrm{G}$, which characterizes the trajectory of $X[k]$ yielding $Z[k]=I_{N}$. Substituting into (6) and setting $Z=I_{N}$, we obtain $\Pi(w)=$ $\exp \left(-(F+C)-\operatorname{vec}^{-1}\left(\left[\begin{array}{ll}\left(\bar{G}_{d}+\bar{D}_{d}\right. & \bar{G}_{c}+\bar{D}_{c}\end{array}\right] w\right)\right)$, which has the local expression $\phi^{-1}(\Pi(w))=$ $-(\widetilde{F}+\widetilde{C})-G w$, where $G:=\left[\begin{array}{ll}\widetilde{G}_{d}+\widetilde{D}_{d} & \widetilde{G}_{c}+\widetilde{D}_{c}\end{array}\right]$. For convenience, define $\pi:=\phi^{-1} \circ \Pi$. We make the manifold characterized by $Z=I_{N}$ controlled-invariant by choosing $u=\Psi(w)$ such that if $X=\Pi(w)$, then $X^{+}=\Pi\left(w^{+}\right)$. Setting $x^{+}=\pi\left(w^{+}\right)$and $x=\pi(w)$, we have $-T \widetilde{B} \Psi(w)=$ $T \widetilde{A}+Q w+\pi(w)-\pi\left(w^{+}\right)$, where $Q:=\left[T \widetilde{Q}_{d}+\widetilde{Q}_{c}\right]$, which simplifies to $T \widetilde{A}+\left(Q+G\left(S-I_{r_{d}+r_{c}}\right)\right) w$, which yields $\Psi(w)=-\frac{1}{T} \widetilde{B}^{\dagger}\left(T \widetilde{A}+\left(Q+G\left(S-I_{r_{d}+r_{c}}\right)\right) w\right)$.

The map $\Psi$ contains the term $-\widetilde{B}^{\dagger} \widetilde{A}$, which will cancel the affine term in the sampled plant dynamics (4). As will be seen in the proof of the following theorem, the remaining dynamics after application of $\Psi$ are linear, thus, we augment $\Psi$ with a stabilizing linear term.

The state-tracking error $E:=X \Pi(w)^{-1}$ is of critical importance in the context of the regulator problem.

Theorem VI.1. If $\operatorname{span}_{\mathbb{F}}\left\{B_{1}, \ldots, B_{m}\right\}=\mathfrak{g}$, then there exists $K \in \mathbb{F}^{m \times n}$ such that

$$
u=K \phi^{-1}\left(X \Pi(w)^{-1}\right)+\Psi(w)
$$

solves the regulator problem with full information.

Proof. Using $e:=\phi^{-1}(E)=x+\widetilde{F}+\widetilde{C}+G w$,

$$
\begin{aligned}
e^{+} & =T \widetilde{A}+T \widetilde{B}(K e+\Psi(w))+Q w+(e+\pi(w))-\pi\left(w^{+}\right) \\
& =\left(I_{n}+T \widetilde{B} K\right) e .
\end{aligned}
$$

By Propositions V.2 and V.3, if $\operatorname{span}_{\mathbb{F}}\left\{B_{1}, \ldots, B_{m}\right\}=\mathfrak{g}$, then there exists a $K$ such that $I_{n}+T \widetilde{B} K$ is Schur.

From Theorem VI.1, it follows that there exists a positively invariant neighbourhood of the identity in $\mathrm{G}$ for suitable $K$. Thus, for $E[0]$ suitably close to the identity, the closed-loop dynamics are well-defined for all $k \geq 0$. Note that there is no restriction on the plant state $X$.

\section{B. Regulation with Output Feedback}

Theorem VI.2 (Separation Principle). If the control law (12) is implemented using the state estimates provided by the state estimator (10), then the closed-loop tracking and estimation errors are locally asymptotically stable.

Proof. Using $\hat{x}=e_{x}+x=e_{x}+e+\pi(w)$,

$$
\begin{aligned}
e^{+}= & T \widetilde{A}+T \widetilde{B}(K(\hat{x}-\pi(\hat{w}))+\Psi(\hat{w}))+Q w+x-\pi\left(w^{+}\right) \\
= & \left(I_{n}+T \widetilde{B} K\right) e+T \widetilde{B} K e_{x} \\
& \quad+T \widetilde{B} K\left(G-Q-G\left(S-I_{r_{d}+r_{c}}\right)\right) e_{w} .
\end{aligned}
$$

Since $\frac{\partial}{\partial e_{x}} e^{+}=I_{N}+T \widetilde{B} K$ and the state estimator dynamics (10) do not depend on $u$, the set of eigenvalues of the closed-loop system using output feedback is the union of the eigenvalues of the state estimator with the eigenvalues of the closed-loop system using state feedback: $\sigma\left(I_{n}+T \widetilde{B} K\right) \sqcup \sigma\left(A_{o}+L C_{o}\right)$.

Corollary VI.3. If $\widehat{Y}, \widehat{X}, \hat{w}:=\left[\begin{array}{ll}\hat{w}_{d}^{\top} & \hat{w}_{c}^{\top}\end{array}\right]^{\top}$, are as defined in (10), and $\operatorname{span}_{\mathbb{F}}\left\{B_{1}, \ldots, B_{m}\right\}=\mathfrak{g}$, then $u=$ $K \phi^{-1}\left(\widehat{X} \Pi(\hat{w})^{-1}\right)+\Psi(\hat{w})$, where $K \in \mathbb{F}^{m \times n}$ satisfies $(12)$, solves the local regulator problem with output information.

\section{Simulation ON $\mathrm{G} \cong \mathrm{SO}(2) \times \mathbb{R}$}

We consider the generalized cylinder $\mathrm{G} \cong \mathrm{SO}(2) \times \mathbb{R}$, with Lie algebra $\mathfrak{g}$. A commuting basis for $\mathfrak{g}$ is

$$
H_{1}=\left[\begin{array}{cc}
0 & -1 \\
1 & 0
\end{array}\right], \quad H_{2}=\left[\begin{array}{ll}
1 & 0 \\
0 & 1
\end{array}\right] \text {. }
$$

We consider a system with exosystem parameters:

$$
S_{d}=\left[\begin{array}{ll}
1 & 1 \\
0 & 1
\end{array}\right], \quad S_{c}=\left[\begin{array}{cc}
0 & -1 \\
1 & 0
\end{array}\right],
$$

which define a ramp and sinusoid, respectively; plant parameters: $A=H_{1}-H_{2}, B_{1}=H_{1}, B_{2}=H_{2}, Q_{d 1}=H_{1}+2 H_{2}$, $Q_{d 2}=-H_{1}+H_{2}, Q_{c 1}=H_{1}+H_{2}, Q_{c 2}=H_{2}$; output parameters: $C=H_{1}+2 H_{2}, D_{d 1}=H_{1}-H_{2}, D_{d 2}=$ $H_{1}+1 H_{2}, D_{c 1}=H_{1}, D_{c 2}=2 H_{1}+H_{2}$; and regulation quantity parameters: $F=H_{1}-3 H_{2}, G_{d 1}=H_{1}+2 H_{2}$, $G_{d 2}=2 H_{1}+H_{2}, G_{c 1}=1 H_{1}-H_{2}, G_{c 2}=3 H_{1}+H_{2}$.

We solve the linear quadratic regulator problem with identity weight matrices to design both our locally stabilizing controller gain $K$ and our state estimator gain $L$. This yields $K=-0.618 I_{2}$ and

$$
\begin{aligned}
L_{1} & =\left[\begin{array}{cc}
-0.855 & 0.165 \\
-0.242 & -2.43
\end{array}\right], \quad L_{2}=\left[\begin{array}{cc}
0.0817 & -0.707 \\
0.0464 & -0.188
\end{array}\right], \\
L_{3} & =\left[\begin{array}{cc}
-0.0942 & -0.00470 \\
-0.256 & 0.111
\end{array}\right] .
\end{aligned}
$$

We use a sampling period of $T=1$ and initialize with $X(0)=\exp \left(0.2 H_{1}+0.2 H_{2}\right), w_{d}[0]=\left[\begin{array}{ll}0 & 0.5\end{array}\right]^{\top}, w_{c}(0)=$ $\left[\begin{array}{ll}0.3 & -0.3\end{array}\right]^{\top}, \widehat{X}[0]=I_{2}, \hat{w}_{d}[0]=\mathbf{0}_{2}, \hat{w}_{c}[0]=\mathbf{0}_{2}$.

Figure 2 depicts the plant states. In Figure 3, we see that $Z[k] \rightarrow I_{N}$, i.e., we have solved the regulator problem. However, we see that $Z(t), t \in(k T,(k+1) T)$ does not tend to $I_{N}$ - there are no guarantees regarding intersample behaviour. In Figure 2, we see that the state on SO(2) (locally) "wraps around" several times, which is characteristic of dynamics on a quotient space.

Since the output is a static map of the states, it too exhibits this wrapping behaviour. However, as depicted in Figure 4, the state estimates converge.

\section{Closing Remark And Future Research}

It is worth noting that all our results hold globally if $G$ has no compact subgroups, i.e., for $\mathrm{G}$ commutative, $\mathrm{SO}(2)$ is not a subgroup of $\mathrm{G}$. Future work includes extending our results to dynamics on nilpotent and solvable Lie groups, and allowing $X, Y$, and $Z$ to be defined on distinct Lie groups. 


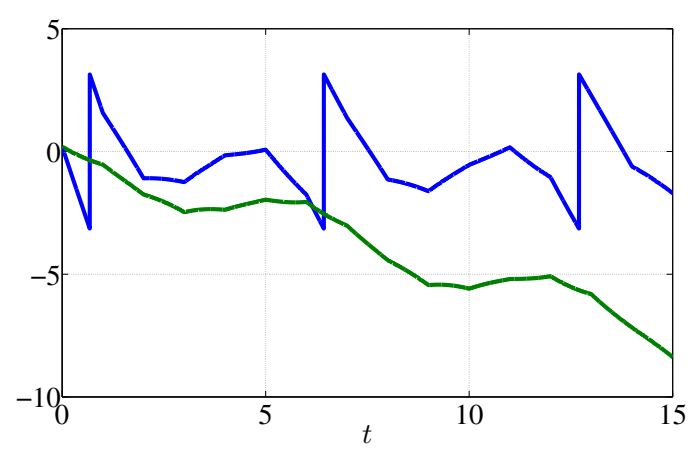

Fig. 2: Plant states $\phi^{-1}(X)$.

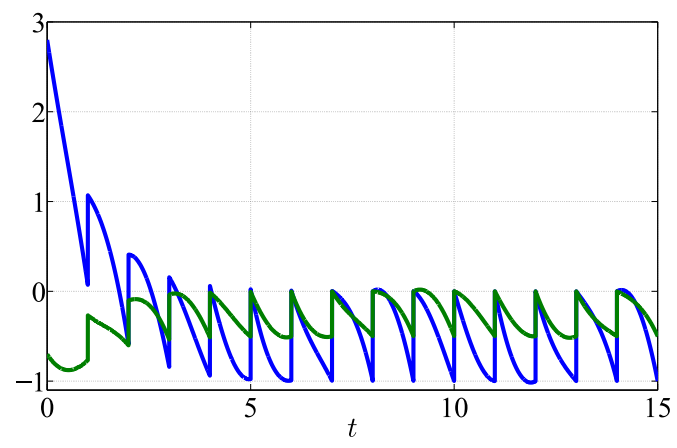

Fig. 3: Regulation quantity $\phi^{-1}(Z)$.

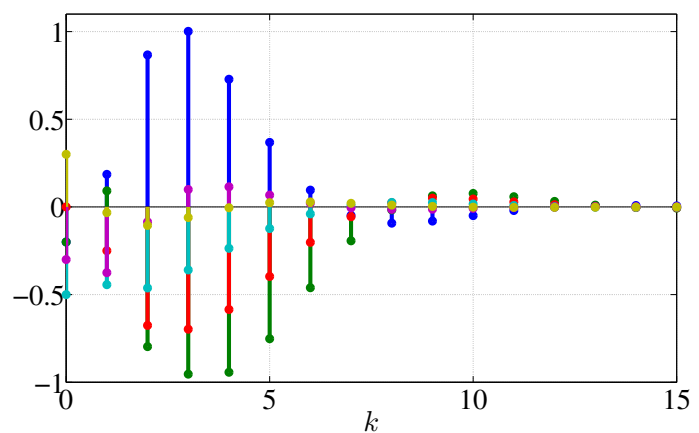

Fig. 4: Estimation errors $\phi^{-1}\left(\widehat{X} X^{-1}\right)$ and $\hat{w}-w$.

\section{REFERENCES}

[1] B. A. Francis, "The Linear Multivariable Regulator Problem," SIAM Journal on Control and Optimization, vol. 15, no. 3, pp. 486-505, 1977. 1

[2] A. Isidori and C. I. Byrnes, "Output regulation of nonlinear systems," IEEE Transactions on Automatic Control, vol. 35, no. 2, pp. 131-140, 1990. 1

[3] B. Castillo, S. Di Gennaro, S. Monaco, and D. Normand-Cyrot, "Nonlinear regulation for a class of discrete-time systems," Systems \& Control Letters, vol. 20, no. 1, pp. 57-65, 1993. 1

[4] G. S. Schmidt, C. Ebenbauer, and F. Allgöwer, "Output Regulation for Control Systems on SE(n): A Separation Principle Based Approach," IEEE Transactions on Automatic Control, vol. 59, no. 11, pp. 30573062, 2014. 1

[5] S. de Marco, L. Marconi, R. Mahony, and T. Hamel, "Output regulation for systems on matrix Lie-groups," Automatica, vol. 87, pp. 8-16, 2018. 1

[6] F. Dörfler and F. Bullo, "Synchronization in complex networks of phase oscillators: A survey," Automatica, vol. 50, no. 6, pp. 15391564, 2014. 1, 2
[7] N. E. Leonard, "Stability of a bottom-heavy underwater vehicle," Automatica, vol. 33, no. 3, pp. 331-346, 1997. 1

[8] T. Lee, M. Leok, and N. H. McClamroch, "Geometric tracking control of a quadrotor UAV on SE(3)," in IEEE Conference on Decision and Control, Atlanta, GA, 2010, pp. 5420-5425. 1

[9] J. Jin, A. Green, and N. Gans, "A stable switched-system approach to obstacle avoidance for mobile robots in SE(2)," in International Conference on Intelligent Robots and Systems, Chicago, IL, 2014, pp. 1533-1539. 1

[10] I. Petersen and D. Dong, "Quantum control theory and applications: a survey," IET Control Theory \& Applications, vol. 4, no. 12, pp. 2651-2671, 2010. 1

[11] A. S. Willsky and S. I. Marcus, "Analysis of bilinear noise models in circuits and devices," Journal of the Franklin Institute, vol. 301, no. 1-2, pp. 103-122, 1976. 1

[12] J. Park and K. Kim, "Tracking on Lie group for robot manipulators," in International Conference on Ubiquitous Robots and Ambient Intelligence. Kuala Lumpur: IEEE, 2014, pp. 579-584. 1

[13] J. R. Forbes, "Passivity-Based Attitude Control on the Special Orthogonal Group of Rigid-Body Rotations," Journal of Guidance, Control, and Dynamics, vol. 36, no. 6, pp. 1596-1605, 2013. 1

[14] O. Egeland and J.-M. Godhavn, "Passivity-based adaptive attitude control of a rigid spacecraft," IEEE Transactions on Automatic Control, vol. 39 , no. 4 , pp. $842-846,1994.1$

[15] G. C. Goodwin, J. C. Agüero, M. E. Cea Garridos, M. E. Salgado, and J. I. Yuz, "Sampling and Sampled-Data Models: The Interface Between the Continuous World and Digital Algorithms," IEEE Control Systems, vol. 33, no. 5, pp. 34-53, 2013. 1

[16] D. Nešić and A. R. Teel, "A Framework for Stabilization of Nonlinear Sampled-Data Systems Based on Their Approximate Discrete-Time Models," IEEE Transactions on Automatic Control, vol. 49, no. 7, pp. 1103-1122, 2004. 1

[17] Z. Mahboubi, Z. Kolter, T. Wang, and G. Bower, "Camera Based Localization for Autonomous UAV Formation Flight," in Infotech@Aerospace. St. Louis, Missouri: American Institute of Aeronautics and Astronautics, 2011. 1

[18] D. Elliott, Bilinear Control Systems - Matrices in Action. SpringerVerlag, 2009. 1

[19] P. J. McCarthy and C. Nielsen, "Passivity-Based Control of SampledData Systems on Lie Groups with Linear Outputs," in Symposium on Nonlinear Control Systems, vol. 49, no. 18, Monterey, CA, 2016, pp. 1006-1011. 1

[20] — - "Local synchronization of sampled-data systems on oneparameter Lie subgroups," in American Control Conference, Seattle, WA, 2017, pp. 3914-3919. 1

[21] S. Monaco and M.-D. Normand-Cyrot, "Accessibility under multirate control for nilpotent lie algebra," in IEEE Conference on Decision and Control, no. 1, 2007, pp. 6262-6267. 1

[22] S. Monaco, D. Normand-Cyrot, and P. Di Giamberardino, "Piecewise constant control of dynamics evolving on Lie groups: a simple case study," in International Confrence on System, Identication and Control Problems, Moscow, 2009, pp. 834-847. 1

[23] M. B. Kobilarov and J. E. Marsden, "Discrete Geometric Optimal Control on Lie Groups," IEEE Transactions on Robotics, vol. 27, no. 4, pp. $641-655,2011.1$

[24] E. D. Sontag, "An eigenvalue condition for sampled weak controllability of bilinear systems," Systems \& Control Letters, vol. 7, no. 4, pp. 313-315, 1986. 1

[25] K. S. Phogat, D. Chatterjee, and R. N. Banavar, "Discrete-time maximum principle on matrix lie groups," CoRR, vol. abs/1612.08022, 2016. [Online]. Available: http://arxiv.org/abs/1612.08022 2

[26] W. M. Wonham, Linear Multivariable Control: a Geometric Approach. New York, NY: Springer, 1979. 2

[27] N. J. Higham, Functions of Matrices. Society for Industrial and Applied Mathematics, 2008. 3

[28] V. I. Arnold, Mathematical Methods of Classical Mechanics, 2nd ed., ser. Graduate Texts in Mathematics. Springer New York, 1989. 3

[29] F. Bullo and A. D. Lewis, Geometric Control of Mechanical Systems. Springer Verlag, 2004. 3

[30] S. MacLane and G. Birkhoff, Algebra, 3rd ed. American Mathematical Society, 1999. 3

[31] W. Magnus, "On the exponential solution of differential equations for a linear operator," Communications on Pure and Applied Mathematics, vol. 7, no. 4, pp. 649-673, 1954. 3 